\title{
Intervención participativa en la gestión de riesgos psicosociales
}

\author{
Participatory intervention in the management of \\ psychosocial risks
}

\author{
María Jesús Fernández Escalona', José Francisco Márquez Matito', Fernando Villalobos Antequera' \\ 1. Empresa Malagueña de Transportes. Málaga. España. \\ Correspondencia \\ María Jesús Fernández Escalona \\ Empresa Malagueña de Transportes. Málaga. España. \\ Correo electrónico: smedico@emtsam.es
}

Resumen

La Empresa Malagueña de Transportes establece un modelo de gestión de riesgos psicosociales fundamentado en la integración, participación, mejora continua, autorresponsabilidad y trabajo en equipo. En el cual se garantiza la integración de la gestión de la prevención de riesgos laborales mediante una política común con los Sistemas de Calidad y Medioambiente en un Sistema Integrado de Gestión. Y en el que la participación de los distintos miembros de la organización a través de las distintas comisiones es un pilar básico para el funcionamiento del mismo y logro de los objetivos.

Así mismo se plantea como objetivos estratégicos de la empresa la mejora del Clima Laboral, la reducción del absentismo y la reducción de la accidentabilidad.

Para conseguir estos objetivos se desarrolla un Modelo de Medición Sistemática y continuo de evaluación de riesgos psicosociales que ha demostrado durante diez años ser un método muy válido para la detección precoz de desviaciones y para la gestión eficaz de este tipo de riesgos.

La implantación del Plan de Comunicación Vertical y Horizontal, junto con el Plan de Formación y la creación de las distintas Comisiones de Trabajo han sido elementos facilitadores para conseguir la participación e implicación activa de todo el personal de la empresa y con ello la asunción de autorresponsabilidad y mejora continua, que queda evidenciada en las medidas adoptadas y la eficacia de los resultados.

Palabras clave: Liderazgo participativo, gestión integrada, riesgos psicosociales, mejora continua.

Abstract

Malaga Transport Company down a psychosocial risk management based on integration, participation, continuous improvement, self-reliance and teamwork. In which ensures the integration of the management of occupational safety through a common policy with Quality and Environmental Systems in Integrated Management System. And in which the participation of the various members of the organization through the various committees is a basic pillar for the operation thereof and achievement of objectives.

It also arises as the company's strategic objectives the improvement of employee, reduced absenteeism and accident reduction.

To achieve these objectives we develop a model Systematic and continuous measurement of psychosocial risk assessment for ten years has proven to be a very valid method for early detection of deviations and to the effective management of these risks. 
The implementation of the Communication Plan Vertical and Horizontal, along with the Training Plan and the creation of the various working committees have been enablers for participation and active involvement of all staff of the company and thus the assumption of self-responsibility and continuous improvement, which is evidenced by the measures adopted and the effectiveness of the results.

Key words: Participative leadership, integrated management, psychosocial risks, continuous improvement. 


\section{INTRODUCCIÓN}

La Empresa Malagueña de Transportes, Sociedad Anónima Municipal, en adelante también EMT, con capital social perteneciente al Excmo. Ayuntamiento de Málaga, desarrolla las siguientes actividades:

- Gestión, explotación y administración de los servicios públicos de los transportes colectivos urbanos.

- Colaboración y participación en la coordinación y/o gestión del transporte colectivo de viajeros en ámbito supramunicipal.

- Gestión, explotación y administración de estaciones cuya gestión directa tenga encomendado el Excmo. Ayuntamiento.

La EMT cuenta con cinco centros de trabajo fijos y 250 centros móviles, autobuses, ubicados en la ciudad de Málaga.

1. Instalaciones principales, donde se encuentra el edificio de administración, servicio de prevención propio, taller de reparación y mantenimiento de la flota de autobuses y aparcamientos.

2. Centro de Atención al cliente.

3. Caseta de Control de la Alameda.

4. Caseta de Control del Parque.

5. Estación de Autobuses en Paseo de los Tilos.

La plantilla de la EMT es de 805 personas, con la siguiente distribución: Administración 48, Movimiento 640 (620 conductores de autobús y 20 inspectores), Taller 106 y Estación de autobuses 11.

Identificación de necesidades en materia de PRL y forma de afrontar la Prevención de Riesgos Laborales por la empresa.

- Los objetivos de la EMT en materia de seguridad y salud forman parte del Plan Estratégico de la empresa. En este se incluye los objetivos de reducción del absentismo, mejora del clima laboral, mejora de las condiciones de trabajo y reducción de la accidentabilidad. En materia de vigilancia de la salud se están implantando programas de promoción de la salud dentro del concepto de Empresa Saludable.

- El Sistema de Gestión de Prevención de Riesgos Laborales en la EMT se encuentra integrado en un Sistema de Gestión Integrada ( SGI) desde el año 2004, cumpliendo con los requisitos de las normas UNE- EN ISO 9001:2000 de Calidad, UNE-EN ISO 14001:2004 de Medio Ambiente y OHSAS 18001:2007 de Sistemas de Gestión de la Seguridad y Salud en el trabajo.

El SGI está basado en los valores de mejora continua, autorresponsabilidad, prevención, cumplimiento de la legislación y visión económica global. En el que la participación de los distintos miembros de la organización a través de las distintas comisiones es un pilar básico para el funcionamiento del mismo y logro de los objetivos.

Para desarrollar las funciones preventivas la empresa cuenta con un Servicio de Prevención Propio con las cuatro especialidades (Medicina del Trabajo, Seguridad en el Trabajo, Higiene Industrial, Ergonomía y Psicosociología) y con un Comité de Seguridad y Salud.

\section{PROBLEMA (PELIGRO/RIESGO/RESULTADO)}

Tras una primera evaluación de riesgos psicosociales en la que se incluyó el análisis de los datos de absentismo, accidentabilidad y estudios epidemiológicos del área de vigilancia de la salud del Servicio de Prevención Propio de la empresa, se planteó la 
necesidad de establecer mejoras a nivel organizativo, de liderazgo, de asunción de responsabilidades, de comunicación, etc.

Para ello se implantó un modelo de gestión de riesgos psicosociales participativa basada en la mejora continua, autorresponsabilidad y el trabajo en equipo, facilitada por la implantación de un sistema de gestión de seguridad y salud certificado en OHSAS en un Sistema Integrado de Gestión y según modelo de Excelencia EFQM.

A su vez se estableció un Modelo de Medición Sistemática y continuo de evaluación de riesgos psicosociales de todos los trabajadores utilizando técnicas combinadas cuantitativas y cualitativas (entrevistas individuales y en grupo), generales y específicas, (aprobadas por los miembros del Comité de Seguridad y Salud). Este método ha demostrado durante estos diez años ser muy válido para la detección precoz de desviaciones y para la gestión eficaz de los riesgos psicosociales en nuestra empresa.

De estas evaluaciones se derivan estudios específicos en las distintas comisiones: de horarios de lineas, turnos de descansos, organización de servicios especiales, etc. en las que participan los representantes de los trabajadores, adoptándose las medidas de mejora necesarias.

\section{MEDIDAS DE PREVENCIÓN DEL RIESGO ADOPTADAS}

\section{Medidas de prevención primaria}

\subsection{Reorganización del trabajo}

\subsubsection{SELECCIÓN DEL PERSONAL}

Recogido en Convenio Colectivo y en el procedimiento del SIG de selección de personal.

Destacamos:

Bolsa de trabajo común. Peón Especializado y Agente único.

El Agente único antes de ser contratado como conductor de autobús de viajero en la vía pública, es contratado como peón especializado en turno nocturno para las funciones de repostado, niveles y limpieza de interior de autobuses. Con ello conseguimos dos objetivos fundamentales en la reducción de riesgos psicosociales: $1^{\circ}$ Que el trabajador aprenda y adquiera destreza en la conducción de los distintos modelos de autobuses antes de salir a la vía pública y transportar viajeros $2^{\circ}$ Disminuir el número de personas fijas en el turno nocturno.

\subsubsection{ROTACIÓN DE PUESTOS DE TRABAJO, REMODELACIÓN Y ENRIQUECIMIENTO DE TAREAS}

- En el turno de noche se ha producido una remodelación de las tareas y gracias a la rotación semanal de los puestos de trabajo (repostado, niveles y limpieza de interior de autobuses) han permitido entre otras mejoras disminuir considerablemente el tiempo de exposición a ruido (producido por la aspiradora en las tareas de limpieza de interior de autobuses).

- En aquellas líneas que circulan por barriadas con mayor riesgo psicosocial, además de estar dotada de vídeo vigilancia en el interior del autobús, también se ha establecido rotación de personal con el fin de que no haya ningún trabajador fijo en la línea a no ser que lo solicite el trabajador.

- En la Estación de Autobuses se ha creado una nueva categoría de ControladorAtención al Cliente al unificar los puestos de Información (con alto riesgo de exigencias psicológicas emocionales: Atención al Público) y controlador de la Estación (riesgo de aislamiento), las rotaciones son semanales. 


\subsection{Clarificación de funciones y competencias}

Recogidas en Convenio Colectivo y en los diversos procedimientos e instrucciones de trabajo.

\subsection{Plan de Comunicación Vertical y Horizontal}

La identificación de nuestras necesidades de comunicación interna se realiza a través de distintos medios:

- Nuestra política de Puertas Abiertas (Accesibilidad a los directivos).

- Los diversos órganos colegiados de participación.

- Las actividades llevadas a cabo por nuestro departamento de Asistencia Social.

- El Buzón de Sugerencias.

- Análisis de no conformidades cuya causa sea la falta de comunicación.

- Comité de Seguridad y Salud.

\begin{tabular}{|c|c|c|}
\hline \multicolumn{2}{|c|}{ Vías de Comunicación } & Frecuencia \\
\hline \multicolumn{2}{|c|}{ Encuesta de clima laboral } & Trianual \\
\hline \multicolumn{2}{|c|}{ Encuesta de prevención } & Anual \\
\hline \multicolumn{2}{|c|}{ Buzón de sugerencias } & Continuo \\
\hline \multicolumn{2}{|c|}{ Sistema de reuniones } & Continuo \\
\hline \multicolumn{2}{|c|}{ Grupos de Mejora } & Anual \\
\hline \multicolumn{2}{|c|}{$\begin{array}{l}\text { Accesibilidad a los directivos «Política de puertas } \\
\text { abiertas" y eliminación de barreras físicas. }\end{array}$} & Continuo \\
\hline QUIÉNES & QUÉ NECESITAN & CÓMO LO HACEMOS \\
\hline Directivos & $\begin{array}{l}\text { - Informe situación puntual } \\
\text { de la Empresa. } \\
\text { - Interrelaciones con las } \\
\text { distintas Áreas. } \\
\text { - Proyectos a medio plazo. }\end{array}$ & $\begin{array}{l}\text { - Comité de Dirección. } \\
\text { - Subcomités Sectoriales. } \\
\text { - Dirección Gerencia. }\end{array}$ \\
\hline Personas & $\begin{array}{l}\text { - Actualidad, cambios y } \\
\text { novedades de la Empresa. } \\
\text { - Receptividad a sus } \\
\text { sugerencias. }\end{array}$ & $\begin{array}{l}\text { - Intranet, Pág. WEB, } \\
\text { Revista EMTRETODOS, } \\
\text { Tablón de anuncios. } \\
\text { - Buzón, Intranet, RRHH. }\end{array}$ \\
\hline
\end{tabular}

\subsection{Trabajo en equipo. Grupos de Mejora}

\begin{tabular}{lcl}
\hline \multicolumn{1}{c}{ GRUPO DE MEJORA } & $\mathbf{N}^{\circ}{ }^{\circ}$ DE PERSONAS & \multicolumn{1}{c}{ OBJETIVOS DEL GRUPO } \\
\hline Movimiento SAE & 12 & $\begin{array}{l}\text { Grupo de coordinación de actividades (horario de } \\
\text { servicio, bajas y sustituciones, hoja de ruta) }\end{array}$ \\
$\begin{array}{l}\text { Taller } \\
\text { Taller y movimiento }\end{array}$ & 12 & $\begin{array}{l}\text { Grupo de análisis de coordinación Intradepartamental. } \\
\text { Grupo de coordinación de actividades taller y } \\
\text { movimiento. }\end{array}$ \\
Administración & 12 & $\begin{array}{l}\text { Grupo de coordinación de actividades intra, } \\
\text { interdepartamental. } \\
\text { Solución de la problemática de uso de las rampas para } \\
\text { personal de movilidad reducida en los autobuses. } \\
\text { Grupo de comunicación estratégica: objetivos, visión y } \\
\text { misión, proyectos. }\end{array}$ \\
\hline 12 & 12 &
\end{tabular}




\subsection{Medidas de protección frente a violencia externa}

\subsubsection{MEDIDAS DE PROTECCIÓN AL TRANSPORTE PÚBLICO}

Las mejoras de accesibilidad de las Paradas, el Plan de Carril Bus, La Semaforización preferente y las Relaciones con Policía Municipal y Tráfico han contribuido a mejorar las exigencias psicológicas cuantitativas del puesto de agente único por la distribución irregular de las tareas producidas por el tráfico así como la reducción de situaciones de violencia externa.

\subsubsection{SISTEMAS DE VIDEO VIGILANCIA}

\subsubsection{PEDAL DE EMERGENCIA}

\subsection{Medidas de ordenación del tiempo de trabajo}

\section{GESTIÓN DE HORARIOS Y SERVICIOS. ROTACIONES Y TURNOS DE TRABAJO}

Además de lo recogido en Convenio Colectivo y en procedimientos del SIG (Diseño y planificación de Líneas, Planificación anual del Servicio, Control de Líneas a través del SAE, Control e inspección de Líneas, Planificación del Servicio Diario etc.), en la EMT se han creado distintas comisiones para tratar estos temas en las que participan trabajadores y personal de la dirección.

\begin{tabular}{|c|c|c|}
\hline Comisiones de Gestión & Actividades & Líderes implicados \\
\hline Comité de Dirección & $\begin{array}{l}\text { Seguimiento semanal, mensual y } \\
\text { anual de indicadores de procesos, } \\
\text { estado de desarrollo de proyectos } \\
\text { y análisis situación económica. }\end{array}$ & Directivos y técnicos específicos. \\
\hline Comité de Seguridad y Salud & $\begin{array}{l}\text { Seguimiento actividad } \\
\text { preventiva, seguimiento de la } \\
\text { accidentabilidad y colaboración } \\
\text { en el desarrollo de los planes de } \\
\text { prevención. }\end{array}$ & $\begin{array}{l}\text { Servicio de prevención, dirección } \\
\text { y agentes sociales. }\end{array}$ \\
\hline Comisión social & $\begin{array}{l}\text { Organización y seguimiento de } \\
\text { actividades sociales dirigidas a } \\
\text { personal, familiares y personal } \\
\text { jubilado. }\end{array}$ & $\begin{array}{l}\text { Trabajador social, área de } \\
\text { personal y agentes sociales. }\end{array}$ \\
\hline Comisiones de procesos & $\begin{array}{l}\text { Coordinación y seguimiento de } \\
\text { la implantación de proyectos } \\
\text { y adaptación en los diferentes } \\
\text { procesos. }\end{array}$ & $\begin{array}{l}\text { Dirección, mandos intermedios y } \\
\text { agentes sociales. }\end{array}$ \\
\hline Comité de igualdad & $\begin{array}{l}\text { Coordinación y seguimiento de } \\
\text { las acciones de mejora en materia } \\
\text { de igualdad del propio plan de } \\
\text { igualdad implantado. }\end{array}$ & $\begin{array}{l}\text { Miembro de la comisión } \\
\text { (representantes de los } \\
\text { trabajadores y dirección). }\end{array}$ \\
\hline
\end{tabular}

\subsection{Medidas especiales para empresas en procesos de reestructuración}

\subsubsection{MEJORA DEL CLIMA LABORAL}

La Mejora del Clima Laboral es un objetivo estratégico en la empresa desde el año 2004 encaminado a mejorar los índices de satisfacción de las personas que integran la EMT. Tras la evaluación de los riesgos psicosociales se adoptaron los siguientes programas:

- Plan de comunicación vertical y horizontal.

- Plan de reconocimientos en toda la empresa. 
- Formación de grupos de mejora.

- Plan anual de formación.

- Plan 23: Reordenación de líneas y turnos.

\subsubsection{RECOLOCACIÓN DE TRABAJADORES CON PROBLEMAS DE SALUD}

\subsubsection{PLAN DE IGUALDAD Y MEDIDAS DE CONCILIACIÓN VIDA LABORAL Y FAMILIAR}

Se han adoptados entre otras medidas:

- Creación de una Comisión de Igualdad.

- Elaboración del Plan de Igualdad.

- Formación en Sensibilización de Género.

- Protocolo de Prevención y Actuación del Acoso Moral, Sexual y por Razón de Sexo.

- Medidas de conciliación vida laboral y familiar.

\section{Medidas de intervención o secundarias}

\subsection{Acciones de Formación y Sensibilización}

Plan de Formación: Analizadas las necesidades se han impartido formación de:

- Atención al Cliente y Control del Estrés.

- Mejora de las Habilidades Directivas de Directivos y Mandos Intermedios. Formación en Competencias directivas, Trabajo en equipo, Motivación, Liderazgo, Negociación, Técnicas de resolución de conflictos, etc.

- Prevención de Riesgos Laborales.

- Sensibilización en Género.

- Gestión del tiempo etc.

\subsection{Protocolo de Prevención y Actuación del Acoso Moral, Sexual y por Razón de Sexo de la Empresa Malagueña de Transportes}

La Dirección de la Empresa y la Representación de los trabajadores y trabajadoras, conscientes de que las conductas de acoso perjudican y repercuten en todo el personal, no sólo en el directamente afectado, se comprometen a prevenir los comportamientos constitutivos de acoso y afrontar las denuncias que puedan producirse por medio del presente Protocolo, basado en los principios de celeridad, protección de derecho a la intimidad y confidencialidad.

\subsection{Procedimientos ante situaciones de violencia externa o de terceros}

Las situaciones de violencia externa o de terceros que causan lesión al trabajador ya sean psíquicas o físicas son registradas e investigadas por la Comisión de Accidentes (Personal del Servicio de Prevención Propio y Delegados de Prevención) según procedimiento de Investigación de Accidentes. Tras la investigación de lo sucedido el trabajador es remitido al departamento jurídico de la empresa para que inicie los trámites legales correspondientes. Si es necesario se procede al cambio de puesto de trabajo o de línea.

\section{Medidas de protección terciarias}

\section{Vigilancia de la salud}

En la empresa tenemos un procedimiento sobre Vigilancia de la Salud y en aquellos casos en los que tras la valoración del examen de salud el médico del trabajo considera 
que el trabajador es Apto con limitaciones, el trabajador es considerado personal sensible y se comunica a los jefes y superiores cuales son las medidas a adoptar para que se proceda a la adaptación del puesto a la persona.

La Vigilancia de la Salud se realiza en el Servicio Médico de la empresa con personal propio. Los exámenes de salud se realizan al ingreso, de forma periódica una vez al año, tras retorno al trabajo tras un periodo superior a un mes de baja por enfermedad y tras cambio de puesto de trabajo.

\section{Empresa saludable}

Se han implantado programas de promoción de la salud para cambios de hábitos de vida saludable.

\section{EFICACIA DE LOS RESULTADOS}

Los indicadores que nos ayudan a medir la eficacia de los resultados son:

Indicadores de Clima Laboral: Encuesta Clima Laboral y Encuesta de Satisfacción Laboral del Servicio de Prevención Propio.

Indicadores de rendimiento: Índice de absentismo, Accidentes de trabajo, Formación, Ascensos, promociones, reconocimientos y generación de empleo, Comunicación interna y Gestión del conocimiento, Actuaciones de la actividad social de la EMT, Vehículos adaptados a Personas de Movilidad Reducida, Grado de cumplimiento de la oferta, Grado de cumplimiento de los horarios.

Los beneficios humanos, sociales y económicos en relación al coste son claramente positivos: reducción del 53\% del absentismo por enfermedad, disminución del 35\% del índice de incidencia de accidentes de trabajo, 95\% de satisfacción laboral con la empresa.

\section{FACTORES DEL ÉXITO}

Liderazgo de la dirección: Integración del Sistema de Gestión de la Seguridad y Salud en el trabajo en un Sistema integrado de Gestión. Certificación OHSAS. Auditorías anuales.

Establecer la Mejora del Clima laboral como un objetivo estratégico de la empresa. Desarrollo del Plan de Comunicación Vertical y Horizontal. Plan de Formación y Trabajo en Equipo.

Participación e implicación activa de los trabajadores en las distintas comisiones. Asunción de autorresponsabilidad y mejora continua.

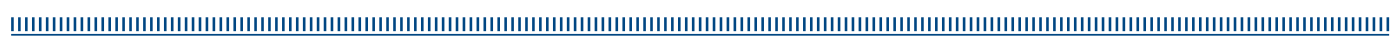

